\title{
GENERALIZED p-FORMS ELECTRODYNAMICS IN CLIFFORD SPACES
}

\author{
C. Castro \\ Center for Theoretical Studies of Physical Systems \\ Clark Atlanta University, Atlanta, Georgia 30314, USA
}

April, 2003 . Revised November, 2003

\begin{abstract}
Using Clifford algebraic methods we describe how to generalize Maxwell theory of Electrodynamics asociated with ordinary point-charges to a generalized Maxwell theory in Clifford spaces involving extended charges and p-forms of arbitrary rank. Clifford algebras contain the appropriate algebraic-geometric features to implement the principle of polydimensional transformations (branes of different dimensionality are rotated into each other) that could possibly lead to a proper formulation of string and $\mathrm{M}$ theory.
\end{abstract}

\section{Introduction : Clifford Algebras in Physics}

Clifford algebras display a rich mathematical structure which is very useful for a unified description of geometry and physics $[1,2,3,4,5,6,9]$. For example, a geometric approach to the physics of the Standard Model in terms of Clifford algebras was advanced by [3] . Important applications have also been found in string theory, extended objects, gravity, QFT.... Using Clifford-algebraic methods, and related ideas, the bosonic $p$-brane propagator, in the quenched-reduced minisuperspace approximation, was constructed in [10]; the logarithmic corrections to the black hole entropy based on the geometry of Clifford space were obtained in [12]; a higher derivative gravity action withtorsion was derived from a geometric action starting from the scalar curvature in Clifford-spaces [11]. Many recent important aspects of Clifford algebra are described in the monographs [5].

In $[7,8,9]$ it was proposed that every physical quantity could in fact be assigned to a polyvector, or multivector, that is aClifford number or aClifford aggregate. Since spinors are the members of left or right minimal ideals of Clifford algebras, this fact may provide the framework for a deeper understanding of sypersymmetries, i.e., the transformations relating bosons and fermions. The Fock-Stueckelberg theory of relativistic particle can also be embedded in the Clifford algebra of spacetime [ 9 ] .

In this new physical theory the arena for physics is no longer the ordinary spacetime, but a more general Clifford manifold of Clifford algebra valued objects, polyvectors. Such a manifold has been called a pandimensional continuum [8] or $C$-space [7]. The latter describes on a unified basis the objects of various dimensionality: not only points, but also closed lines, surfaces, volumes,.., called 0-loops (points), 1-loops (closed strings) 2-loops (closed membranes), 3-loops, etc.. It is a sort of a dimension category, where the role of functorial maps is played by C-space transformations which reshuffle a $p$-brane history for a $p^{\prime}$-brane history or a mixture of all of them, for example.

Technically those transformations in C-space that reshuffle objects of different dimensions are generalizations of the ordinary Lorentz transformations, that mix space with time, to $C$-space. Since Clifford algebras contain the appropriate algebraic-geometric features to implement this principle of polydimensional transformations and are deeply ingrained in the algebraic properties of quaternions and octonions [17], it is very plausible that this may lead to the proper formulation of string and M theory [11] within the framework of Projective Geometry [18] .

The main purpose of this letter is to describe how to generalize Maxwells theory of Electrodynamics asociated with ordinary point-charges to a Generalized Maxwell theory in Clifford spaces involving extended charges and p-forms of arbitrary rank. Clifford algebras implement naturally the idea of electric-magnetic duality and incoporate Dirac monopoles in a straightforwad fashion [ 13 ]. Other recent approaches to the generalization of Maxwell's Electrodynamics has been based in the study of gerbes or higher-gauge theories [15]. For a detailed discussion of the role of Clifford algebras and Electrodynamics see $[4,14]$.

\subsection{Extending Relativity from Minkowski spacetime to $C$-space}


In order to generalize Maxwell theory of Electrodynamics we review briefly the extended relativity theory in C-spaces $[7,9,11]$ by starting with a natural generalization of the notion of a space-time interval in Minkowski space $d s^{2}$ to C-space. This is given by the scalar part of the Clifford geometric product of two infinitesimal displacements :

$$
d X^{2}=<d X^{+} d X>_{\text {scalar }}=d \Omega^{2}+d x_{\mu} d x^{\mu}+d x_{\mu \nu} d x^{\mu \nu}+\ldots
$$

where the operation $X^{+}$is the analog of the matrix transpose ( hermitian ) conjugation operation and represents the reversed Clifford multivector obtained by reversing the ordering position of all the basis vectors $\gamma^{\mu}$ present in the expansion of $X$.

The Clifford valued multivector:

$$
X=X^{M} E_{M}=\Omega I+x^{\mu} \gamma_{\mu}+x^{\mu \nu} \gamma_{\mu} \wedge \gamma_{\nu}+\ldots
$$

denotes the position of a polyparticle in a manifold, called Clifford space or $C$-space.

The coordinates $x^{\mu_{1} \mu_{2}}, x^{\mu_{1} \mu_{2} \mu_{3}}, \ldots$ are the holographic areas, volumes, ...proyections of the nested family of $p$-loops onto the embedding spacetime coordinate planes/hyperplanes .In order to match units in the expansion of the multivector $X$ it is required to introduce a length scale. Such scale was chosen to be the minimal Planck scale $[7,16]$ and can be set to unity.

For example, if we take differential $d X$ of $X$ and compute the scalar product $\langle d X d X\rangle$ we obtain the C-space analog of the particle proper time interval :

$$
d \Sigma^{2}=(d \Omega)^{2}+\Lambda_{P}^{2 D-2} d x_{\mu} d x^{\mu}+\Lambda_{P}^{2 D-4} d x_{\mu \nu} d x^{\mu \nu}+.
$$

where in (1-3) one has introduced explicitly the Planck scale $\Lambda_{P}$ in order to fuse together objects of different dimensionality : 0-loops ( points ), 1-loops ( closed strings ), ..,p-loops ( closed p-branes ). Einstein introduced the speed of light as a universal absolute invariant in order to "unite" space with time (to match units) in the Minkwoski space interval:

$$
d s^{2}=c^{2} d t^{2}-d x_{i} d x^{i}
$$

A similar unification is needed here to "unite" objects of different dimensions, such as $x^{\mu}, x^{\mu_{1} \mu_{2}}$, etc... The minimal Planck scale then emerges as another universal invariant in constructing an extended scale relativity theory in C-spaces $[7,16]$.

To continue along the same path of special Relativity in Minkowski space, one considers the analog of Lorentz transformations in C-spaces which transform a polyvector $X$ into another polyvector $X^{\prime}$ given by $X^{\prime}=R X R^{-1}$ with

$$
R=e^{\theta^{A} E_{A}}=\exp \left[\left(\theta I+\theta^{\mu} \gamma_{\mu}+\theta^{\mu_{1} \mu_{2}} \gamma_{\mu_{1}} \wedge \gamma_{\mu_{2}} \ldots .\right)\right]
$$

and

$$
R^{-1}=e^{-\theta^{A} E_{A}}=\exp \left[-\left(\theta I+\theta^{\nu} \gamma_{\nu}+\theta^{\nu_{1} \nu_{2}} \gamma_{\nu_{1}} \wedge \gamma_{\nu_{2}} \ldots . .\right)\right] . \quad R R^{-1}=1
$$

where the theta parameters:

$$
\theta ; \theta^{\mu} ; \theta^{\mu \nu} ; \ldots
$$

are the C-space version of the Lorentz rotations/boosts parameters.

Since a Clifford algebra admits a matrix representation, one can write the norm of a polyvectors in terms of the trace operation as: $\|X\|^{2}=$ Trace $X^{2}$ Hence under C-space Lorentz transformation the norms of poly-vectors behave like follows:

$$
\text { Trace } X^{\prime 2}=\text { Trace }\left[R X^{2} R^{-1}\right]=\text { Trace }\left[R R^{-1} X^{2}\right]=\text { Trace } X^{2} \text {. }
$$

These norms are invariant under C-space Lorentz transformations due to the cyclic property of the trace operation and $R R^{-1}=1$. This completes the very brief review of relativity in C-spaces [ 7, 9, 11$]$. 


\section{C-space Generalized Maxwell Electrodynamics}

In this section we will generalize Maxwell Electrodynamics to C-spaces ; i.e. construct the Clifford algebra-valued extension of the Abelian field strength $F=d A$ associated with ordinary vectors $A_{\mu}$. Based on the standard definition of the Abelian field strength $F=d A$ we shall use the same definition in terms of polyvector-valued quantities and differential operators in C-space :

$$
A=A_{N} E^{N}=\Phi I+A_{\mu} \gamma^{\mu}+A_{\mu \nu} \gamma^{\mu} \wedge \gamma^{\nu}+\ldots \ldots
$$

the first component in the expansion $\Phi$ is a scalar field; $A_{\mu}$ is the standard Maxwell field $A_{\mu}$, the third component $A_{\mu \nu}$ is a rank two antisymmetric tensor field.....and the last component of the expansion is a pseudo-scalar. The fact that a scalar and pseudo-scalar field appear very naturally in the expansion of the C-space polyvector valued field $A_{N}$ suggests that one could attempt to identify the latter fields with the dilaton and the axion field, respectively. Once again, in order to match units in the expansion ( 21 ), it requires the introduction of suitable powers of a length scale parameter, the Planck scale which is conveniently set to unity.

The differential operator is the generalized Dirac operator :

$$
d=E^{M} \partial_{M}=I \partial_{\Omega}+\gamma^{\mu} \partial_{x_{\mu}}+\gamma^{\mu} \wedge \gamma^{\nu} \partial_{x_{\mu \nu}}+\ldots
$$

the polvector valued indices $M, N \ldots$ range from $1,2 \ldots .2^{D}$ since a Clifford algebra in $D$-dim has $2^{D}$ basis elements. At this point we will not be concerned with the representations of Clifford algebras in $(r, s)$ dimensions. Where $r, s$ denotes the temporal and spatial dimensions respectively [ 2 ] .

The generalized Maxwell field strength in C-space is :

$$
\begin{gathered}
F=d A=E^{M} \partial_{M}\left(E^{N} A_{N}\right)=E^{M} E^{N} \partial_{M} A_{N}=\frac{1}{2}\left\{E^{M}, E^{N}\right\} \partial_{M} A_{N}+ \\
\frac{1}{2}\left[E^{M}, E^{N}\right] \partial_{M} A_{N}=\frac{1}{2} F_{(M N)}\left\{E^{M}, E^{N}\right\}+\frac{1}{2} F_{[M N]}\left[E^{M}, E^{N}\right] .
\end{gathered}
$$

where one has decomposed the Field strength components into a symmetric plus antisymmetric piece by simply writing the Clifford geometric product of two polyvectors $E^{M} E^{N}$ as the sum of an anticommutator plus a commutator piece respectively .

$$
\begin{aligned}
& F_{(M N)}=\frac{1}{2}\left(\partial_{M} A_{N}+\partial_{N} A_{M}\right) . \\
& F_{[M N]}=\frac{1}{2}\left(\partial_{M} A_{N}-\partial_{N} A_{M}\right) .
\end{aligned}
$$

The standard Maxwell action :

$$
S(A)=\int[\mathcal{D} X] F_{[M N]} F^{[M N]} .
$$

for the measure in C-space :

$$
[\mathcal{D} X]=(d \Omega)\left(d x^{0} d x^{1} \ldots d x^{D}\right)\left(d x^{01} d x^{02} \ldots\right)\left(d x^{012} \ldots\right) \ldots \ldots .\left(d x^{0123 \ldots D}\right) .
$$

is automatically gauge invariant under the transformations $\delta A_{M}=\partial_{M} \Lambda$ since $\delta F_{[M N]}=0$ due to the antisymmetry condition $\left[\partial_{M}, \partial_{N}\right] \Lambda=0$.

We will show below how the most general C-space Maxwell action is up to a numerical factor given by :

$$
\int[\mathcal{D} X]\left\langle F^{\dagger} F>_{\text {scalar }}=\int[\mathcal{D} X]\left(F_{(M N)} F^{(M N)}+F_{[M N]} F^{[M N]}\right) .\right.
$$

is invariant ( up to total derivatives) under infinitesimal gauge transformations provided the symmetric part of $F$ is divergence-free $\partial_{M} F^{(M N)}=0$. 
It was later noticed in [19] that this divergence-free condition has the same effects as if one were fixing a gauge leaving a residual symmetry of restricted gauge transformations such that the gauge symmetry parameter obeys the Laplace-like equation $\partial_{M} \partial^{M} \Lambda=0$. Such residual ( restricted ) symmetries are precisely those that leave invariant the divergence-free condition on the symmetric part of $F$. Residual, restricted symmetries occur, for example, in the light-cone gauge of p-brane actions leaving a residual symmetry of volume-preserving diffs and in string theory, in the conformal gauge, leaving a residual symmetry under conformal reparametrizations; i.e. the Virasoro algebras whose symmetry transformations are given by holomorphic and anti-holomorphic reparametrizations of the string world-sheet.

This Laplace-like condition on the gauge parameter is also the one required such that the action (2-7) is invariant under finite (restricted) gauge transformations [19] since under such (restricted) finite transformations the Lagrangian (2-7) changes by second-order terms of the form $\left(\partial_{M} \partial_{N} \Lambda\right)^{2}$, which are total derivatives if, and only if, the gauge parameter is restricted to obey the analog of Laplace equation $\partial_{M} \partial^{M} \Lambda=0$ [19]. Concluding, the effects of adding the symmetric pieces of $F$ to the action (2-7) leads to a restricted gauge symmetry which has the same effects as if one were fixing a gauge $\partial_{M} F^{(M N)}=0[19]$.

The matter-field minimal coupling is :

$$
\int A_{M} d X^{M}=\int J_{M} A^{M}
$$

where one has reabsorbed the coupling constant, the C-space analog of the electric charge, within the expression for the $A$ field itself . Notice that this term ( 2-8 ) has the same form as the coupling of p-branes ( whose world volume is $p+1$-dimensional ) to antisymmetric tensor fields of rank $p+1$.

The open line integral in C-space of the matter-field interaction term in the action ( 2-8 ) is taken from the polyparticles proper time interval $\Sigma$ ranging from $-\infty$ to $+\infty$ and can be recast via the Stokes law solely in terms of the antisymmetric part of the field strength. This requires closing off the integration countour by a semi-circle that starts at $\Sigma=+\infty$, goes all the way to C-space infinity, and comes back to the point $\Sigma=-\infty$. The field strength vanishes along the points of the semi-circle at infinity, and for this reason the net contribution to the contour integral is given by the open-line integral. Therefore, by rewriting the $\int A_{M} d X^{M}$ via the Stokes law relation it yields :

$$
\begin{aligned}
\int A_{M} d X^{M}= & \int F_{[M N]} d \Sigma^{[M N]}=\int F_{[M N]} X^{M} d X^{N}= \\
& \int d \Sigma F_{[M N]} X^{M}\left(d X^{N} / d \Sigma\right) .
\end{aligned}
$$

where in order to go from the second term to the third term in the above equation ( 2-9) we have integrated by parts and then used the Bianchi identity for the antisymmetric component $F_{[M N]}$ :

$$
\begin{gathered}
\partial_{L} F_{[M N]}+\partial_{M} F_{[N L]}+\partial_{N} F_{[L M]}= \\
\partial_{L}\left(\partial_{M} A_{N}-\partial_{N} A_{M}\right)+\partial_{M}\left(\partial_{N} A_{L}-\partial_{L} A_{N}\right)+\partial_{N}\left(\partial_{L} A_{M}-\partial_{M} A_{L}\right)=0 .
\end{gathered}
$$

The integration by parts ( 2-10) permits us to go from a C-space domain integral represented by the Clifford-value hypersurface $\Sigma^{M N}$, to a C-space boundary-line integral :

$$
\int d \Sigma^{M N}=\frac{1}{2} \int\left(X^{M} d X^{N}-X^{N} d X^{M}\right)
$$

The pure matter terms in the action are given by the analog of the proper time integral spanned by the motion of a particle in spacetime :

$$
\kappa \int d \Sigma=\kappa \int d \Sigma \sqrt{\frac{d X^{M}}{d \Sigma} \frac{d X_{M}}{d \Sigma}} .
$$

where $\kappa$ is a parameter whose dimensions are $(\text { mass })^{p+1}$ and $\Sigma$ is the polyparticle proper time in C-space ( 1-3 ) . The Lorentz force relation in C-space is directly obtained from a variation of 


$$
\int d \Sigma F_{[M N]} X^{M}\left(d X^{N} / d \Sigma\right) .
$$

and

$$
\kappa \int d \Sigma=\kappa \int \sqrt{d X^{M} d X_{M}} .
$$

with respect to the $X^{M}$ variables :

$$
\kappa \frac{d^{2} X_{M}}{d \Sigma^{2}}=e F_{[M N]} \frac{d X^{N}}{d \Sigma} .
$$

where we have re-introduced the $\mathrm{C}$-space charge $e$ back into the Lorentz force equation in C-space. A variation of the terms ( 2-7, 2-8) of the action w.r.t the $A_{M}$ field furnishes the following equation of motion for the $A$ fields :

$$
\partial_{M} F^{(M N)}+\partial_{M} F^{[M N]}=J^{N} .
$$

Gauge invariance will impose further restrictions on the symmetric part $F^{(M N)}$. The gauge variation of the symmetric part $F_{(M N)}$ is not zero but equals:

$$
\delta F_{(M N)}=\partial_{M} \partial_{N} \Lambda \neq 0
$$

since the gauge variation of the fields is given by :

$$
\delta A_{M}=\partial_{M} \Lambda .
$$

where $\Lambda$ is a C-space scalar-valued parameter. One can notice that the gauge variation of $F_{[M N]}=0$ since

$$
\left[\partial_{M}, \partial_{N}\right] \Lambda=0 .
$$

As we said above, the action (2-7 ) would be gauge invariant if, and only if, one sets the following condition :

$$
\partial_{M} F^{(M N)}=0 .
$$

which leads to a restricted gauge-invariance of the moste general action ( 2-7 ) compared to the ordinary unrestricted gauge-invaraince of the Maxwell action involving only the antisymmetric components $F_{[M N]}$.

If this condition ( 2-20) is imposed then the restricted gauge variation of ( 2-7) yields :

$$
\begin{aligned}
\delta \int[\mathcal{D} X] F_{M N} F^{M N} & =\delta \int[\mathcal{D} X]\left(F_{(M N)} F^{(M N)}+F_{[M N]} F^{[M N]}\right)= \\
& 2 \int[\mathcal{D} X] F^{(M N)} \partial_{M} \partial_{N} \Lambda .
\end{aligned}
$$

since the gauge variation of $F^{[M N]}=0$. If we integrate by parts the last term of $(2-21)$ we get for the gauge variation of the Maxwell term :

$$
2 \int[\mathcal{D} X] \partial_{M}\left[F^{(M N)} \partial_{N} \Lambda\right]-2 \int[\mathcal{D} X]\left(\partial_{N} \Lambda\right)\left(\partial_{M} F^{(M N)}\right) .
$$

If the are no boundary terms and/or if the fields vanish fast enough at infinity the total derivative terms are zero and the first terms of the gauge variation is zero. Hence, the C-space generalized Maxwell action ( 2-7 ) is invariant under the ( restricted ) gauge transformations provided the symmetric component of the field strength is divergence-free : $\partial_{M} F^{(M N)}=0$. This condition is also compatible with the field equations of the gauge fields. After breaking the field equations into a symmetric and antisymmetric component yields after implementing the condition ( 2-20) : 


$$
\partial_{M} F^{(M N)}=0
$$

and

$$
\partial_{M} F^{[M N]}=J^{N} .
$$

Finally, by taking derivatives on both sides of the last equation with respect to the $X^{N}$ coordinate , one obtains due to the symmmetry condition of $\partial_{M} \partial_{N}$ versus the antisymmetry of $F^{[M N]}$ that :

$$
\partial_{N} \partial_{M} F^{[M N]}=0=\partial_{N} J^{N}=0 .
$$

which is precisely the continuity equation for the current.

Therefore we have found that a restricted gauge invariance of the action ( 2-7 ) demands that $\partial_{M} F^{(M N)}$ 0 , which is indeed compatible with the continuity equation for the current. The continuity equation is essential to ensure that the matter-field coupling term of the action $\int A_{M} d X^{M}=\int J_{M} A^{M}$ is also gauge invariant, which can be readily verified after an integration by parts and setting the boundary terms to zero

$$
\delta \int[\mathcal{D} X] J^{M} A_{M}=\int[\mathcal{D} X] J^{M} \partial_{M} \Lambda=-\int[\mathcal{D} X]\left(\partial_{M} J^{M}\right) \Lambda=0 .
$$

Gauge invariance also ensures the conservation of the energy-momentum (via Noethers theorem ) defined in tems of the Lagrangian density variation as [13]:

$$
T^{M N}=\left(\partial^{M} A_{K}\right)\left(\frac{\delta \mathcal{L}}{\delta\left(\partial_{N} A_{K}\right)}\right)-G^{M N} \mathcal{L} .
$$

where $G^{M N}$ is the C-space metric ( in this case flat ). The canonical conjugate momenta for the field $A$ is :

$$
\pi_{N M}=\partial_{N} A_{M}
$$

It remains to be seen if this construction of C-space generalized Maxwell Electrodynamics of p-forms can be generalized to the Nonabelian case when we replace ordinary derivatives by gauge-covariant ones :

$$
F=d A \rightarrow F=D A=(d A+A \bullet A) .
$$

For example, one could define the graded-symmetric product $E_{M} \bullet E_{N}$ based on the graded commutator of Superalgebras:

$$
[A, B]=A B-(-1)^{s_{A} s_{B}} B A .
$$

$s_{A}, s_{B}$ is the grade of $A$ and $B$ respectively. For bosons the grade is even and for fermions is odd. In this fashion the graded commutator captures both the anti-commutator of two fermions and the commutator of two bosons in one stroke. Using ( 2-31) one may extend this definition to :

$$
E_{M} \bullet E_{N}=E_{M} E_{N}-(-1)^{s_{M} s_{N}} E_{N} E_{M} .
$$

$s_{M}, s_{N}$ is the grade of $E_{M}$ and $E_{N}$ respectively. Even or odd depending on the grade of the basis elements.

One may generalize Maxwells theory to that of Born-Infeld nonlinear Electrodynamics in C-spaces based on our construction presented in this letter. Finally, the extension from a flat C-space to a curved $\mathrm{C}$-space also warrants further investigation in order to couple C-space Yang-Mills theory to C-space gravity ( a higher derivative gravity with torsion [11] ). This will be left for a future publication.

\section{Acknowledgements}

We are indebted to Matej Pavsic for numerous discussions which led to this work and to Frank ( Tony ) Smith and M. Bowers for their invaluable help.

\section{References}

1-D. Hestenes, "Spacetime Algebra" Gordon and Breach, New York, 1996. 
D. Hestenes and G. Sobcyk, "Clifford Algebra to Geometric Calculus" D. Reidel Publishing Company, Dordrecht, 1984.

2-I. R. Porteous, Clifford Algebras and the Classical Groups , Cambridge University Press, 1995.

3-W. Baylis, Electrodynamics, A Modern Geometric Approach , Boston, Birkhauser, 1999.

G. Trayling and W. Baylis, A Geometric Approach for the Standard Model hep-th/0103137.

4-B. Jancewicz, Multivectors and Clifford Algebra in Electrodynamics World Scientific, Singapore 1989.

5- "Clifford Algebras and their applications in Mathematical Physics" Vol 1: Algebras and Physics. eds by R. Ablamowicz, B. Fauser. Vol 2: Clifford analysis. eds by J. Ryan, W. Sprosig Birkhauser, Boston 2000.

6-P. Lounesto, "Clifford Algebras and Spinors". Cambridge University Press. 1997.

7-C. Castro, Chaos, Solitons and Fractals 11 (2000) 1663. Foundations of Physics 30 (2000) 1301. Chaos, Solitons and Fractals 11 (2000) 1721. Chaos, Solitons and Fractals 12 (2001) 1585. The Programs of the Extended Relavity in C-spaces, towards physical foundations of String Theory Advance NATO Workshop on the Nature of Time, Geometry and the Physics of Perception. Kluwer Publishers 2003.

8-W. Pezzaglia, "Physical Applications of a Generalized Geometric Calculus" gr-qc/9710027. Dimensionally Democratic calculus and Principles of Polydimensional Physics gr-qc/9912025. Classification of Multivector Theories and Modifications of the Postulates of Physics gr-qc/9306006.

9-M.Pavsic: "The Landscape of Theoretical Physics": A Global View Kluwer, Dordrecht 1993. Foundations of Physics 31 (2001) 1185. Phys. Let A 242 (1998) 187. Nuovo Cimento A 110 (1997) 369. Clifford Space as the Arena for Physics gr-qc/0211085, to appaer in Foundations of Physics ( 2003 ).

10- S. Ansoldi, A. Aurilia, C. Castro, E. Spallucci, Phys. Rev. D 64026003 (2001)

11-C. Castro, M. Pavsic : Phys. Lets B 539 ( 2002 ) 133.

12-C. Castro, Jour. of Entropy 3 (2001) 12-26.

13-M. Defaria-Rosa, E. Recami and W. Rodriguez: Phys. Letts B 173 ( 1986 ) 233.

14-T. Ivezic, Foundations of Physics Lett 15 ( 2002 ) 27. Invariant Relativistic Electrodynamics, Clifford Algebra Approach hep-th/0207250.

15-H. Pfeiffer : Higher Gauge Theoy and Nonabelian generalization of 2-form Electrodynamics hepth/0304074. J. Baez: Higher Yang-Mills theory hep-th/0206130.

16- L. Nottale : Fractal Spacetime and Microphysics, towards the Theory of Scale Relativity World Scientific 1992.

17- P. Ramond, Exceptional Groups and Physics hep-th/0301050.

18- D. Hestenes and R. Ziegler : Projective Geometry with Clifford Algebra , Acta Applicandae Mathematicae 23 ( 1991 ) 25-63.

19-C. Castro, M.Pavsic, The Extended Relativity Theory in Clifford Spaces to appear in the Int.Jour.Mod.Phys. A. 